\title{
LA VIVIENDA Y LA EXPLOTACIÓN AGRARIA EN GALICIA. UN SISTEMA INTEGRADO PARA EL ESTUDIO DE CALIDAD DE VIDA
}

\author{
(HOUSING AND FARMING IN GALICIA. AN INTEGRATED SYSTEM \\ FOR STUDIES OF QUALITY OF LIFE)
}

Florentino Díaz Rodríguez y Carlos J. Álvarez López, Dres. Ingenieros Agrónomos Dpto. de Ingeniería Agroforestal. Univ. de Santiago de Compostela

ESPAÑA

Fecha de recepción: 23-IV-98

\section{RESUMEN \\ Estudio de un sistema formado por la interacción de la explotación de ganado vacuno, muy representativa del sector agrario de Galicia y la vivienda familiar vinculada a ésta. \\ Se analizaron 94 variables estudiadas sobre una base de 103 explotaciones, encontrándose 4 componentes principales representativos del sistema.}

Esto ha permitido establecer una tipología de las explotaciones ganaderas de Galicia en función de parámetros de calidad de vida, determinándose cuales son realmente los factores de diseño que afectan a la misma.

\section{SUMMARY}

This is a study a system formed by the interaction of cow cattle farming, a very representative activity in Galicia (Spain), an the farmer's house linked to the farm.

94 variables have been analysed in a set of 103 farms, and 4 representative principal components of the system have been found.

This has alloved to find different categories of cattle farming in Galicia according to parameters of quality of life and defining which are the real design factors that affect quality.

\section{INTRODUCCIÓN. EL CONCEPTO DE CALIDAD DE VIDA}

El contacto con las gentes de las áreas rurales gallegas, la actividad profesional en el campo de los proyectos agrarios y las largas reflexiones que han tenido lugar sobre el concepto de calidad de vida, han dado lugar a la idea, a partir de la cual, se ha llevado a cabo este trabajo.

Generalmente se asocia el término calidad de vida con diversas situaciones y circunstancias que afectan a la vida de los individuos; así, por ejemplo, es frecuente hacer referencia a salud, condiciones higiénico-sanitarias, confort ambiental en la vivienda, disponibilidad de infraestructuras, oferta cultural, educación pública y otras. Por ello, resultaría muy difícil encontrar una fórmula que permita, de forma objetiva, calcular la calidad de vida que posee un determinado grupo de personas.

Se trata de un término que actualmente se utiliza de forma generalizada y, en la mayor parte de las ocasiones, con un significado impreciso. Así, por ejemplo: (a) Para hacer referencia a situaciones específicas: tratamientos terapéuticos que mejoran la calidad de vida de pacientes; un tipo de ventanas que mejoran la calidad de vida en la vivienda, por sus buenas condiciones de aislamiento térmico; un electrodoméstico que mejora la calidad de vida de las amas de casa; etc. Por ello, entendemos que la primera característica del concepto es la de un fuerte grado de imprecisión.

(b) En otras ocasiones el término es utilizado para representar un índice del bienestar general, en cuyo caso se trata de encontrar unos valores que suelen estar en función de aspectos como renta per cápita, mortalidad infantil, dotación de infraestructuras, etc. en cuyo caso se pueden obtener unos valores con un mayor grado de objetividad. Este tipo de indicadores se utilizan, sobre todo, para evaluar el nivel de desarrollo en países atrasados.

(c) Para establecer los objetivos a alcanzar en los programas, planes y proyectos de desarrollo rural aunque, desgraciadamente, lo más frecuente es que esos objetivos no se detallen de forma concreta y la calidad de vida a alcanzar se vea reducida a los resultados que se puedan 
derivar de la realización de los diferentes proyectos. En este sentido, el concepto se limita a plantear, como objetivo general, la mejora de las características de los factores existentes con influencia sobre el bienestar de la población.

Ambigüedad e imprecisión son aspectos que hay que eliminar en los trabajos, tanto científicos como técnicos $\mathrm{y}$, por ello, es por lo que planteamos como idea inicial la de buscar un método que permita calcular un valor representativo de calidad de vida en el ámbito rural y, en particular, en la Comunidad Autónoma de Galicia.

La dificultad que ello entraña no es la misma que la que se presenta para evaluar calidad de vida, por poner un ejemplo, la de los enfermos de cáncer de próstata, pues, en este caso, las variables a estudiar se limitan a determinadas constantes vitales del enfermo, mientras que la calidad de vida de las familias puede estar representada por un gran número de variables de muy diversa naturaleza ya que, por otra parte, para que exista calidad de vida se requiere que todas ellas alcancen unos umbrales mínimos, bastando que una sola de ellas obtenga un valor inferior para que el resultado final no sea representativo.

Como se deduce de lo expresado anteriormente, para que calidad de vida tenga un significado concreto es indispensable:

. Establecer el ámbito territorial o marco geográfico del estudio.

. Definir el elemento o elementos a estudiar o valorar, las condiciones en las que se desarrolla y los elementos con los que interacciona.

\section{. Definir los parámetros asociados a calidad de vida.}

Otro factor importante es el conocimiento de las necesidades de las personas, pues, de alguna manera, la satisfacción de éstas condiciona su propia calidad de vida; por ello, el factor humano es un factor imprescindible a tener en cuenta aunque, por otra parte, sus deseos y necesidades concretas no deben ser tenidas en cuenta, para eliminar un importante grado de subjetividad que podría falsear el resultado final del estudio. En este sentido, tal como señalan Doyal y

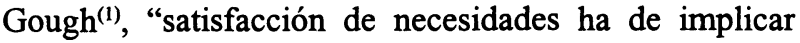
necesariamente indicadores sociales cuantitativos sobre grupos de población" y para evaluar la satisfacción de necesidades debe empezarse por conocer las "características satisfactorias universales" comunes a todas las culturas que son los siguientes:

. Necesidades básicas: salud física y autonomía personal.

. Necesidades intermedias: alimentación, agua potable, alojamiento, peligrosidad en el ambiente de trabajo, entorno físico no peligroso, atención sanitaria, seguridad en la infancia, relaciones de primer grado significativas, seguridad física, seguridad económica, enseñanza, seguridad nacimientos-partos.

\section{. Condiciones sociales previas.}

Hay que considerar que en los países desarrollados, tanto las necesidades básicas como las intermedias y las condiciones sociales previas mínimas están garantizadas, aunque, en determinadas zonas, áreas o grupos sociales pueden encontrarse satisfechas en mayor o menor medida, cuestión que habría que analizar en cada caso.

Por último, señalar lo apuntado por Gómez Orea ${ }^{(2)}$ que admite que "Los problemas y aspiraciones de la sociedad son cambiantes, evolucionan en el tiempo y varían en el espacio, de tal forma que lo que deba entenderse por calidad de vida es función de una serie de factores cuya importancia es también variable", concluyendo que "En esquema, la calidad de vida de un individuo está determinada por la integración de su nivel de renta, condiciones de vida y trabajo y calidad ambiental".

\section{EL ENFOQUE DEL TRABAJO}

Tradicionalmente la calidad de vida, como objetivo de las políticas de las administraciones, se pensó en satisfacerla mediante subvenciones, ayudas a la producción, concentración parcelaria en las áreas rurales, etc., olvidándose de que las condiciones de la vivienda influyen, en gran medida, sobre calidad de vida.

Asimismo, la vivienda rural tradicional, en Galicia, ha sido el núcleo alrededor del cual se han creado la mayor parte de las explotaciones ganaderas, aspecto que condiciona, en gran medida, el estado actual de éstas.

Por otro lado, las explotaciones de ganado vacuno son muy representativas del sector agrario en esta comunidad, con una gran importancia en relación con el conjunto de la actividad socioeconómica, que se deriva de los siguientes aspectos:

. Las producciones ganaderas suponen el 56,77\% de la Producción Final Agraria de Galicia ${ }^{(3)}$, de las cuales el $68 \%$ corresponden al subsector del ganado vacuno.

- La población activa ocupada en el sector agrario es muy elevada, siendo absorbida, en su mayor parte, por las explotaciones de ganado vacuno.

- El subsector ganadero representa, cada vez más, un importante consumidor de productos de la industria química, de maquinaria y equipos y aporta las materias básicas para un floreciente industria agroalimentaria, cada vez más competitiva en el ámbito comunitario 
Las explotaciones ganaderas de Galicia, mayoritariamente, tienen carácter familiar y, por otra parte, poseen también una característica muy especial, como es la de constituir no solamente lugar de trabajo, sino también de residencia familiar. A este respecto conviene tener en cuenta que, a partir de los años 80-90, en Europa se realizan esfuerzos para reducir la producción agraria y, al mismo tiempo, mantener la población en las áreas rurales. Esto significa que en la UE existe una opción política explícita por el mantenimiento de las explotaciones agrarias familiares y que la explotación familiar agraria es la forma mayoritaria de organización social de la agricultura europea, siendo considerada en "su doble vertiente de unidad de convivencia y de producción agraria".

Por otra parte, hay que hacer mención a las referencias básicas, que se derivan de la política comunitaria para el medio rural en el final de siglo, que son las siguientes:

1. Remodelar el uso del espacio, intentando que absorba a parte de la población activa.

2. Mantenimiento de la explotación familiar agraria como organización social dominante en la agricultura.

3. Reducción de la intervención y apoyo público al sector productivo

4. Consideración del agricultor como sujeto de la actividad productiva que desarrolla, de manera simultánea, una actividad de protección del medio ambiente y de desarrollo rural.

5. Las actividades no productivas se pretenden fomentar sobre la base de medidas estructurales en términos de diversificación de la producción, ayudas a actividades no agrarias, a la repoblación forestal y al medio ambiente.

La problemática concreta de la vivienda rural presenta un conjunto de características específicas por el hecho de estar integrada con una explotación agraria y, según el sistema que estudiamos:

- Dependen de factores agrícolas y ganaderos,

- Requieren una superficie elevada,

- Dependen de las características del hábitaty de la población, - Dependen de la organización social de las diferentes áreas, - Uso conjunto del espacio físico por actividades agrarias y vida privada.

Por ello, entendemos que la calidad de vida en el medio rural debe ser evaluada a partir del nivel que poseen las condiciones de vida y trabajo de las personas, considerando que éstas forman parte de un sistema complejo, al que denominaremos Sistema "Vivienda Familiar-Explotación Agraria", sobre el cual inciden diferentes variables que afectan a la calidad de vida.
No se consideran de forma individual ni la renta, ni los factores de producción, infraestructuras o características arquitectónicas de los edificios, sino la consideración global de un Sistema (4), entendido como "un conjunto de elementos interrelacionados entre si y con el medio o entorno que los contiene"

La finalidad de este trabajo se concreta en el estudio del sistema "Vivienda-Explotación Agraria" en Galicia, desde el punto de vista de la calidad de vida que ofrece a los miembros de la familia rural, bajo las siguientes restricciones:

\section{. MARCO GEOGRÁFICO:}

Galicia

\section{. ELEMENTO A ESTUDIAR:}

Sistema vivienda familiar-explotación agraria.

\section{. CRITERIOS A APLICAR:}

Condiciones higiénico-sanitarias y confort ambiental y seguridad de las personas miembros de la unidad familiar dependiente de la explotación.

\section{. PARÁMETROS ASOCIADOS A CALIDAD DE VIDA:}

Se determinarán a partir del presente trabajo.

\section{LOS OBJETIVOS}

Estudiar las variables que inciden sobre calidad de vida en el Sistema, clasificándolas, sistematizándolas y analizando las relaciones entre ellas.

Estudio del Sistema, estableciendo una tipología de las explotaciones agrarias, obteniendo clases homogéneas para las variables de mayor interés.

Establecer los parámetros de diseño, con incidencia sobre condiciones de calidad de vida, a utilizar en los proyectos de explotaciones ganaderas y viviendas rurales asociadas a ellas.

En suma, se plantea modificar los planteamientos clásicos, puramente productivistas, los cuales se ha comprobado que no son plenamente eficaces y que, dadas las orientaciones de las nuevas políticas comunitarias, es preciso reconsiderar.

\section{METODOLOGÍA}

Inicialmente se determinaron las variables representativas que, estimamos, caracterizan adecuadamente cada uno de los componentes del "Sistema". Esto se llevó a cabo 
mediante un proceso de análisis en el cual se identificaron todos los factores, que denominaremos "variables iniciales".

Seguidamente se realizó una encuesta, por muestreo, entre las explotaciones de ganado vacuno de Galicia, en donde se recogieron los valores que presentan dichas variables, lo cual nos permite conocer el estado actual de las mismas.

La información obtenida de la muestra fue objeto de un proceso de sistematización, realizado en tres etapas:

- Selección de las variables iniciales más representativas.

- Representación gráfica de las explotaciones.

- Elaboración de una base de datos con el conjunto de la información.

Posteriormente, se realizó un análisis multidimensional de los datos obtenidos, desarrollado en las siguientes etapas:

1. Eliminación de las variables iniciales redundantes o poco fiables.
2. Aplicación del análisis multivariante, con objeto de encontrar unas nuevas variables, que sean representativas del conjunto del sistema "Vivienda Familiar-Explotación Agraria". Trabajamos con elementos de naturaleza diversa que se integran en un conjunto o sistema y pretendemos encontrar los factores representativos del mismo.

3. Clasificación de las explotaciones estudiadas. Se procederá a clasificar a las explotaciones en función de las nuevas variables de calidad de vida, mediante la utilización de técnicas de análisis multivariante.

\subsection{El material utilizado}

Como se desprende de lo anterior, nuestro material es el "Sistema" formado por vivienda y explotación de ganado vacuno en Galicia, el cual subdividimos en seis componentes básicos (Tabla 1).

Los criterios adoptados para delimitar la "Parcela-base" en los casos en los que su contorno no es plenamente

\section{TABLA 1}

Los componentes del "Sistema"

\begin{tabular}{|c|c|c|}
\hline Factor humano: & \multicolumn{2}{|c|}{$\begin{array}{l}\text { Familia mono o polinuclear dependiente de la explotación. Sujeto activo y } \\
\text { pasivo de sus actividades: Gestión y mano de obra. }\end{array}$} \\
\hline \multirow{3}{*}{$\begin{array}{l}\text { Base territorial: } \\
\text { Conjunto de todas las } \\
\text { fincas y parcelas } \\
\text { dependientes de la } \\
\text { explotación. }\end{array}$} & Finca principal: & $\begin{array}{l}\text { Finca, dentro de la cual se encuentra la vivienda y } \\
\text { otros componentes de la explotación. }\end{array}$ \\
\hline & Parcela-base: & $\begin{array}{l}\text { Parcela en la que está ubicada la vivienda familiar y, } \\
\text { en muchas ocasiones, también las instalaciones } \\
\text { centrales de la explotación. Forma parte de la finca } \\
\text { principal o, en algunos casos, ambas son coinciden- } \\
\text { tes. }\end{array}$ \\
\hline & Otras fincas: & Resto de las fincas dependientes de la explotación. \\
\hline \multirow[t]{2}{*}{ Edificaciones: } & Vivienda y anexos: & $\begin{array}{l}\text { Edificación que está muy vinculada a la explotación, } \\
\text { existiendo una muy estrecha relación entre sí. En la } \\
\text { mayor parte de los casos las actividades privadas y } \\
\text { las agrarias comparten un espacio común. }\end{array}$ \\
\hline & Edificios agrarios: & $\begin{array}{l}\text { La mayor parte de ellos albergan actividades } \\
\text { molestas e insalubres para la vida familiar, } \\
\text { generándose efluentes contaminantes, polvo, malos } \\
\text { olores y riesgo físico para ancianos y niños: } \\
\text { almacenes, establos, silos, heniles, fosa de purín, } \\
\text { sala de ordeño, lechería, parideras, áreas de cría, } \\
\text { etc. }\end{array}$ \\
\hline Ganado: & \multicolumn{2}{|c|}{$\begin{array}{l}\text { Animales que producen purines y estiércoles y que transitan frecuentemente en } \\
\text { el interior de la Parcela-base. }\end{array}$} \\
\hline Instalaciones: & \multicolumn{2}{|c|}{$\begin{array}{l}\text { Instalaciones de ordeño, instalaciones de refrigeración de leche, instalaciones de } \\
\text { limpieza mecanizada, tratamiento de purines, preparación de alimentos para el } \\
\text { ganado, etc. }\end{array}$} \\
\hline Maquinaria: & \multicolumn{2}{|c|}{$\begin{array}{l}\text { Elementos móviles autopropulsados que generan polvo, ruidos y riesgo para las } \\
\text { personas. }\end{array}$} \\
\hline
\end{tabular}


coincidente con los de la "Finca Principal", han sido los siguientes:

- Utilización de los límites físicos que rodean al espacio ocupado por las instalaciones ganaderas y la vivienda con sus anexos.

- Si en alguna de sus partes no existieran, se tomó el criterio de trazar líneas paralelas a las edificaciones y a una distancia de éstas de 5 metros.

\subsection{El muestreo}

Se decidió la realización de una encuesta por muestreo con objeto de conocer el "Sistema" desde dentro, a través del estudio de sus componentes. Las características de la población muestral son:

- Explotaciones de ganado vacuno de Galicia.

- Con carácter familiar.

- Que hayan realizado saneamiento ganadero.

- Que la actividad agraria constituya actividad principal para sus miembros.

Para la elección de la muestra nos hemos basado en el censo elaborado por las Campañas de Saneamiento Ganadero (por ser la única fuente de información fiable)(Tabla 2).
CUADRO 1

\begin{tabular}{|l|c|}
\hline & MUESTRA \\
\hline LA CORUÑA & 33 \\
\hline LUGO & 58 \\
\hline ORENSE & 4 \\
\hline PONTEVEDRA & 8 \\
\hline Tamaño de la muestra & 103 \\
\hline
\end{tabular}

Del total de 150 explotaciones seleccionadas, se pudo materializar, con éxito, el trabajo de recogida de datos en 103 de ellas (Cuadro 1).

\subsection{El método de trabajo}

\subsubsection{Recogida de la información}

Se realizó el diseño de un cuestionario, con objeto de recoger la información de forma ordenada y sistematizada, para lo cual se llevó a cabo un proceso en donde, todos los elementos del sistema, tanto los susceptibles de afectar a las condiciones de calidad de vida como los descriptivos delmismo, se transformaron en variables, unas, cuantitativas

TABLA 2

Explotaciones de vacuno en Galicia

\begin{tabular}{||l|c|c|c|c|c||}
\hline \multicolumn{1}{|c|}{ PROVINCIA } & \multicolumn{2}{c|}{ EXPLOTACIONES } & \multicolumn{2}{c|}{ N$^{\circ}$ DE RESES } & RESES/EXPLOTACIÓN \\
\hline LA CORUÑA & 39.266 & $37,64 \%$ & 419.342 & $38,86 \%$ & 10,68 \\
\hline LUGO & 31.331 & $30,04 \%$ & 477.077 & $44,21 \%$ & 15,23 \\
\hline ORENSE & 11.899 & $11,40 \%$ & 67.947 & $6,29 \%$ & 5,71 \\
\hline PONTEVEDRA & 21.826 & $20,92 \%$ & 114.799 & $10,64 \%$ & 5,26 \\
\hline GALICIA & 104.322 & $100,00 \%$ & 1.079 .165 & $100,00 \%$ & 10,34 \\
\hline
\end{tabular}

Fuente: Campaña de Saneamiento Ganadero. Xunta de Galicia 1994. Elaboración propia.

La estrategia de muestreo estuvo marcada por la carencia de información previa, así se optó por muestrear un gran número de variables en un número relativamente pequeño de explotaciones, pero tratando de maximizar la variabilidad del grupo muestreado. Para ello se seleccionaron, de forma aleatoria, 150 explotaciones, de entre todas las censadas en la Campaña de Saneamiento Ganadero. y otras, cualitativas, denominadas, todas ellas, "variables de trabajo".

Para ello se contó con la opinión experta de técnicos profesionales, tanto de la administración pública como de la empresa privada, que colaboraron en la redacción, tanto del borrador inicial del cuestionario como de la versión 
definitiva del mismo, que se elaboró después de un período de prueba en el que se realizaron algunas encuestas.

Se adoptó el criterio de recogida de información de forma que fuera posible llevar a cabo un análisis gráfico de la misma, ya que el estudio del espacio compartido por vida y trabajo requiere, obviamente, un análisis de ese tipo (Tabla 3).

\subsubsection{Análisis de la información}

El resultado de la encuesta realizada en las explotaciones seleccionadas se introduce en una base de datos, estando representada además, gráficamente, la Parcela-base de cada una de ellas.

En esta etapa de la metodología, el criterio adoptado fue el

TABLA 3

\section{Elementos a encuestar}

\begin{tabular}{|c|c|}
\hline COMPONENTES DEL SISTEMA & FACTORES \\
\hline Identificación & $\begin{array}{l}\text { Nombre de la casa } \\
\text { Identificación catastral }\end{array}$ \\
\hline Localización & $\begin{array}{l}\text { Provincia. Municipio. Parroquia. Lugar. Distancia a capital municipio. } \\
\text { Distancia a capital provincia. Distancia a casa cercana. } \mathrm{N}^{\circ} \text { casas } \\
\text { agrupamiento. Tipo de hábitat. }\end{array}$ \\
\hline Factor humano & $\begin{array}{l}\text { Propietario. Edad. Estado civil. Miembros de la familia. Menores de } 18 \\
\text { años. Jubilados. Percepción de rentas no agrarias. Pertenencia a } \\
\text { Cooperativas. Cursos Capacitación. Estudios Universitarios. }\end{array}$ \\
\hline Base territorial & $\begin{array}{l}\text { Concentración Parcelaria. Superficie total. Número de fincas y/o } \\
\text { parcelas. Superficie forrajera. Superficie labrada. Superficie monte y } \\
\text { forestal. }\end{array}$ \\
\hline La Parcela-base & $\begin{array}{l}\text { Superficie. Superficie construida. Número de edificaciones. Garaje. } \\
\text { Distancia desde vivienda a garaje. Vientos dominantes. Linderos. } \\
\text { Observaciones. }\end{array}$ \\
\hline $\begin{array}{l}\text { La Vivienda en la } \\
\text { Parcela-base }\end{array}$ & $\begin{array}{l}\text { Estado general de conservación. Año construcción. Año última reforma. } \\
\text { Carácter (Aislada o adosada). } \mathrm{N}^{\circ} \text { de plantas. Superficie ocupada. } \\
\text { Superficie construida. Superficie útil. Cuerpos adosados. Retranqueo } \\
\text { vial. Retranqueo linderos. Distribución PB. } \mathrm{N}^{\circ} \text { dormitorios. } \mathrm{N}^{\circ} \\
\text { baños/aseos. } \mathrm{N}^{\circ} \text { total piezas. } \mathrm{N}^{\circ} \text { plazas de garaje. Material estructura. } \\
\text { Forjados. Cubierta. } \mathrm{N}^{\circ} \text { vertientes. Cerramiento. Color cerramiento. } \\
\text { Carpintería exterior. Electricidad. Fontanería. Calefacción. Saneamiento. } \\
\text { Abastecimiento de agua. Tipo de cocina familiar. Trastero. Despensa. } \\
\text { Tendedero. Subvención obras rehabilitación. Equipamiento de la } \\
\text { vivienda. Distancia a fosa de purín. Distancia a establos. Distancia a } \\
\text { silos. Consulta con expertos. }\end{array}$ \\
\hline Edificaciones agrarias & $\begin{array}{l}\text { Capacidad de los alojamientos. Superficie establos. Superficie heniles. } \\
\text { Almacenes. Silos. Sala de ordeño. Lechería. Fosa de purín (tipo, forma, } \\
\text { dimensiones y material). Asesoramiento con expertos. Observaciones. }\end{array}$ \\
\hline Ganado & $\begin{array}{l}N^{0} \text { total de cabezas. } N^{0} \text { vacas leche. } N^{\circ} \text { vacas carne. Producción de } \\
\text { leche. Producción de terneros. }\end{array}$ \\
\hline Instalaciones & $\begin{array}{l}\text { Año de adquisición. características técnicas y estado de conservación } \\
\text { de: Instalación de ordeño. Refrigeración de leche. Limpieza mecanizada. } \\
\text { Equipos informáticos. Preparación y suministro de alimentos. }\end{array}$ \\
\hline Maquinaria & $\begin{array}{l}\text { Nivel de mecanización. Relación de máquinas con: Año de adquisición, } \\
\text { características técnicas y estado de conservación. }\end{array}$ \\
\hline
\end{tabular}


de estudiar más intensamente las características del espacio definido por la Parcela-base, por ser aquél en el cual se producen un mayor número de interferencias entre actividad privada y actividad agraria.

El análisis y estudio de toda la información sirve para crear las variables iniciales del "Sistema", obtenidas a partir de la eliminación de algunas de las "Variables de trabajo", consideradas anteriormente, y de la consideración de otras, que fueron observadas posteriormente en el estudio de la base de datos y representación gráfica de la Parcela-base. Las "variables iniciales del Sistema" son las que servirán para el análisis multidimensional y que se describen seguidamente.

\subsubsection{Las variables iniciales del "Sistema"}

Además de la selección realizada entre las variables de trabajo estudiadas, se introdujeron nuevos factores, derivados del estudio de las interferencias materiales entre las actividades privada y agrarias en la Parcela-base.

Esto llevó consigo un proceso, a partir del que se representa la Parcela-base, en la cual se distinguen cuatro áreas bien diferenciadas:

(a) Área de construcciones ganaderas: se determinará mediante la medición de la superficie ocupada en planta por todas las edificaciones cuya utilidad sea la de albergar las actividades agropecuarias. Aparece representada en la figura 1 , con todas las edificaciones señaladas.

(b) Área privada: sobre el plano se procedió a señalar una línea divisoria que delimita dicha área, que abarca la superficie ocupada por la vivienda y la zona que la rodea, cuya finalidad sea, exclusivamente, usos particulares de la familia y en la cual no se produzca circulación de elementos de la explotación. En la figura $n^{\circ} 1$, se corresponde con la vivienda y un pequeño garaje.

(c) Espacios comunes: es la zona que rodea a la vivienda y en la cual se producen actividades agrarias pero, al mismo tiempo, es el lugar en donde se desarrollan actividades privadas de los miembros de la familia. Zona en la cual el espacio está compartido por actividad privada y agraria. En la figura $\mathrm{n}^{\circ} 1$ es la zona rayada.

(d) Espacios agrarios: es la zona de la parcela-base en la cual se realizan, exclusivamente, actividades agrarias, no afectando, de forma directa, a los quehaceres familiares. Se corresponde con los espacios en blanco, situados entre las edificaciones ganaderas.

Seguidamente se muestra, a modo de ejemplo, la representación de la parcela-base de una de las explotaciones estudiadas.

En el plano de cada una de las explotaciones se representa-

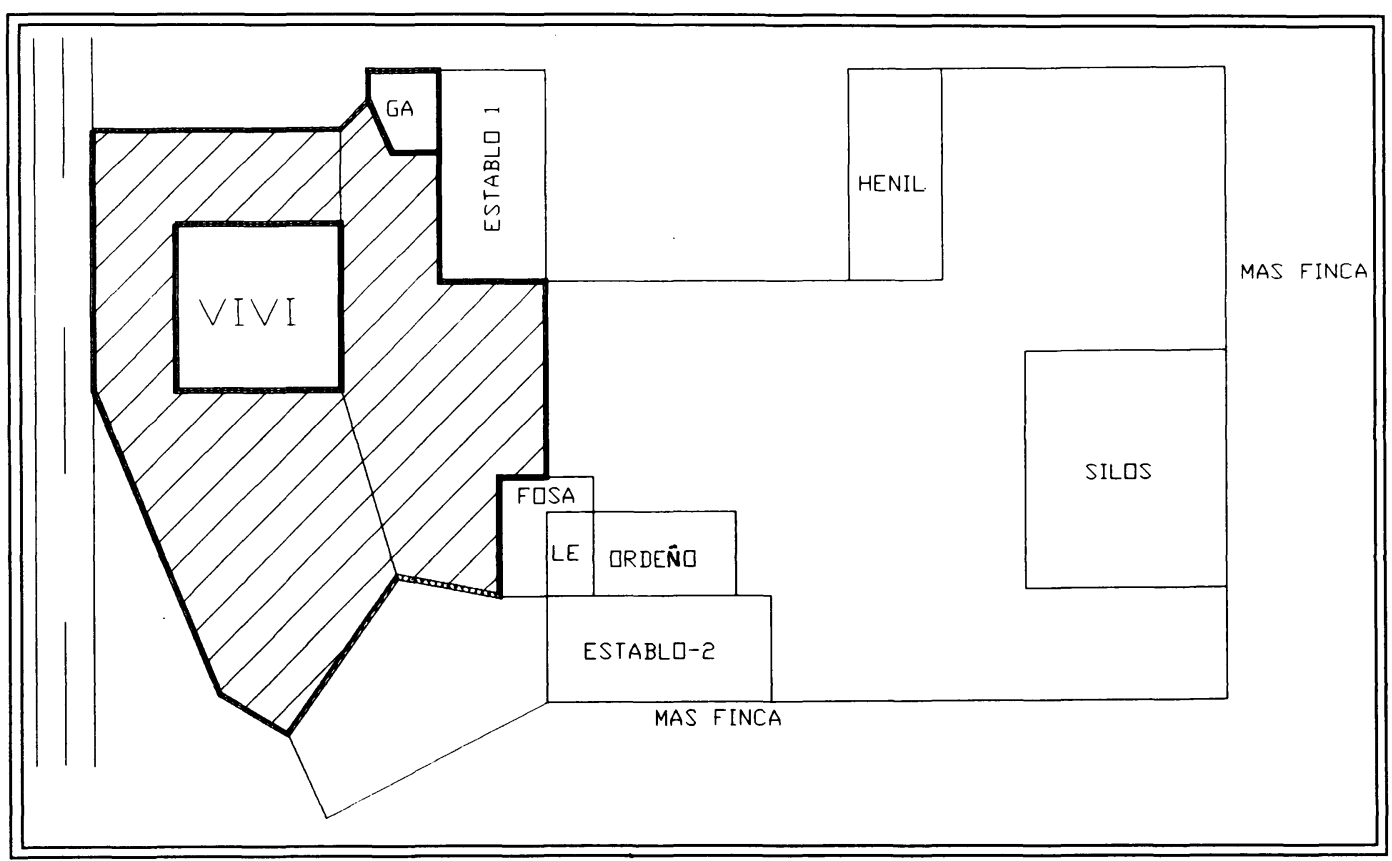

Figura 1.- Áreas de la Parcela-base. 
ron las rutas y circulaciones de los elementos de la explotación agraria, que fueron identificados tal y como se indica en el cuadro 2.

CUADRO 2

\begin{tabular}{||c|l|c||}
\hline 1 & Ganado & $\mathrm{C}$ \\
\hline 2 & $\begin{array}{l}\text { Deyecciones, estiércoles } \mathrm{y} \\
\text { purines }\end{array}$ & $\mathrm{P}$ \\
\hline 3 & Forrajes ensilados & $\mathrm{E}$ \\
\hline 4 & Forrajes en verde & $\mathrm{F}$ \\
\hline 5 & Heno & $\mathrm{H}$ \\
\hline 6 & Maquinaria & $\mathrm{M}$ \\
\hline 7 & Camión recogida de leche & $\mathrm{CA}$ \\
\hline
\end{tabular}

La circulación de estos elementos genera unos efectos, que podemos resumir en:

- Deterioro del firme o pavimento de la zona en la que se producen.

- El pisoteo y tránsito de vehículos en zonas deficientemente pavimentadas, dadas las condiciones climatológicas de Galicia, producen zonas encharcadas, con abundante barro, lo cual es fuente de suciedad en el entorno de la casa.

- El desprendimiento de polvo, partículas de materiales de naturaleza orgánica, como son: hierbas, tierra, polvo, fertilizantes, piensos, deyecciones, productos fermentados, etc., constituyen un caldo de cultivo óptimo para el desarrollo de microorganismos que pueden afectar negativamente a la salud humana.

- El desprendimiento de esas partículas sobre el suelo producen, asimismo, unas condiciones muy favorables para el desarrollo de insectos que, a su vez, producirán efectos poco saludables y muy molestos en el área de influencia de la vivienda.

Con respecto a la selección de las variables iniciales correspondientes al factor de rutas y circulaciones, los criterios adoptados han sido los siguientes:

- Determinación del número de rutas que se producen en el interior de la parcela-base.

- Longitud de cada una de ellas.

- Determinación de la distancia mínima desde cada una de ellas hasta la vivienda.

El valor correspondiente a cada una de ellas se determinó sobre el plano de cada una de las explotaciones, realizando la medición correspondiente.
Las variables de este grupo son las que aparecen en el cuadro 3.

Para ilustrar la forma en la cual se realizó la medición de cada una de las variables se describe, seguidamente, el plano de una de las explotaciones, procediendo a detallar sobre el mismo la medición correspondiente a cada uno de estos parámetros (Figura 2).

Se observa que la circulación de ganado se produce a través de la rutas señaladas con los números 1 y 2 , que se producen desde los dos alojamientos ganaderos existentes hasta el exterior de la parcela. Las vacas en ordeño se encuentran en el "establo-2" y, dado que la sala de ordeño se encuentra adosada al mismo, no se producen otras circulaciones de ganado.

La extracción de purín se realiza a través de la ruta número 3 , desde la fosa de purín hasta el exterior de la parcela.

El tránsito del ensilaje tiene lugar por las rutas números 4 y 5 , desde el lugar en el que se confecciona hasta las dos estabulaciones existentes, donde se distribuye al ganado.

Los forrajes verdes circulan a través de las números 1 , 2 y 6; las dos primeras sirven para distribuir el forraje directamente a los animales en sus estabulaciones y, la tercera, corresponde a la entrada de forraje para ser ensilado.

El heno se introduce en el henil a través de la ruta número 7 distribuyéndose, posteriormente a las estabulaciones a través de las rutas números 8 y 9 .

La circulación de maquinaria se produce a través de las rutas número 8 , desde el almacén en el cual se guardan los aperos hasta el exterior de la parcela.

El camión de recogida de la leche circula desde el vial de acceso hasta la lechería, a través de la ruta número 10 .

Por último, los suministros y servicios de la explotación tienen lugar a través de las rutas números 2 y 10.

La forma de contabilizar el número de rutas es considerando las diferentes circulaciones que se producen por cada ruta; así por ejemplo, la ruta $\mathrm{n}^{\circ} 1$ del ejemplo anterior serviría para la circulación tanto de ganado como de forrajes y la $\mathrm{n}^{\circ} 2$ es utilizada por ganado, forrajes y suministros.

Para determinar la longitud de cada una de ellas se procede a la medición sobre el plano.

Para conocer la distancia desde las rutas hasta la vivienda, se mide sobre el plano la distancia mínima de cada ruta hasta la casa representada en el plano, aspecto que ilustramos en la figura 3. 
CUADRO 3

\begin{tabular}{|c|c|c|}
\hline GRUPOS & & VARIABLES \\
\hline Número de rutas & NRUT: & Número total de rutas \\
\hline GRUPOS & & VARIABLES \\
\hline Longitud de rutas & $\begin{array}{l}\text { LRUT: } \\
\text { LGAN: } \\
\text { LPUR: } \\
\text { LENS: } \\
\text { LFOR: } \\
\text { LHEN: } \\
\text { LMAQ: } \\
\text { LCAM: } \\
\text { LSUM: }\end{array}$ & $\begin{array}{l}\text { Longitud total de las rutas } \\
\text { Longitud rutas de ganado } \\
\text { Longitud rutas de purín } \\
\text { Longitud rutas de ensilado } \\
\text { Longitud rutas de forrajes verdes } \\
\text { Longitud rutas heno } \\
\text { Longitud rutas maquinaria } \\
\text { Longitud ruta camión leche } \\
\text { Longitud rutas suministros y servicios }\end{array}$ \\
\hline GRUPOS & & VARIABLES \\
\hline $\begin{array}{l}\text { Distancia mínima de } \\
\text { rutas a la vivienda }\end{array}$ & $\begin{array}{l}\text { DGAN: } \\
\text { DPUR: } \\
\text { DENS: } \\
\text { DFOR: } \\
\text { DHEN: } \\
\text { DMAQ: } \\
\text { DCAM: } \\
\text { DSUM: }\end{array}$ & $\begin{array}{l}\text { Distancia rutas de ganado } \\
\text { Distancia rutas de purín } \\
\text { Distancia rutas de ensilado } \\
\text { Distancia rutas de forraje } \\
\text { Distancias rutas de heno } \\
\text { Distancia rutas de maquinaria } \\
\text { Distancia rutas camión leche } \\
\text { Distancia rutas suministro y servicos }\end{array}$ \\
\hline GRUPOS & & VARIABLES \\
\hline Otras & $\begin{array}{l}\text { RUTS: } \\
\text { DRUT: } \\
\text { DMIN: } \\
\text { NENTR: } \\
\text { NGANA: }\end{array}$ & $\begin{array}{l}\text { Longitud total de las rutas en función de superficie de Parcela- } \\
\text { base } \\
\text { Distancia media correspondiente a todas las distancias } \\
\text { mínimas } \\
\text { Distancia mínima existente desde una ruta hasta la vivienda } \\
\text { Número de accesos a la Parcela-base } \\
\text { Número de edificaciones dedicadas a alojamiento ganadero. }\end{array}$ \\
\hline
\end{tabular}

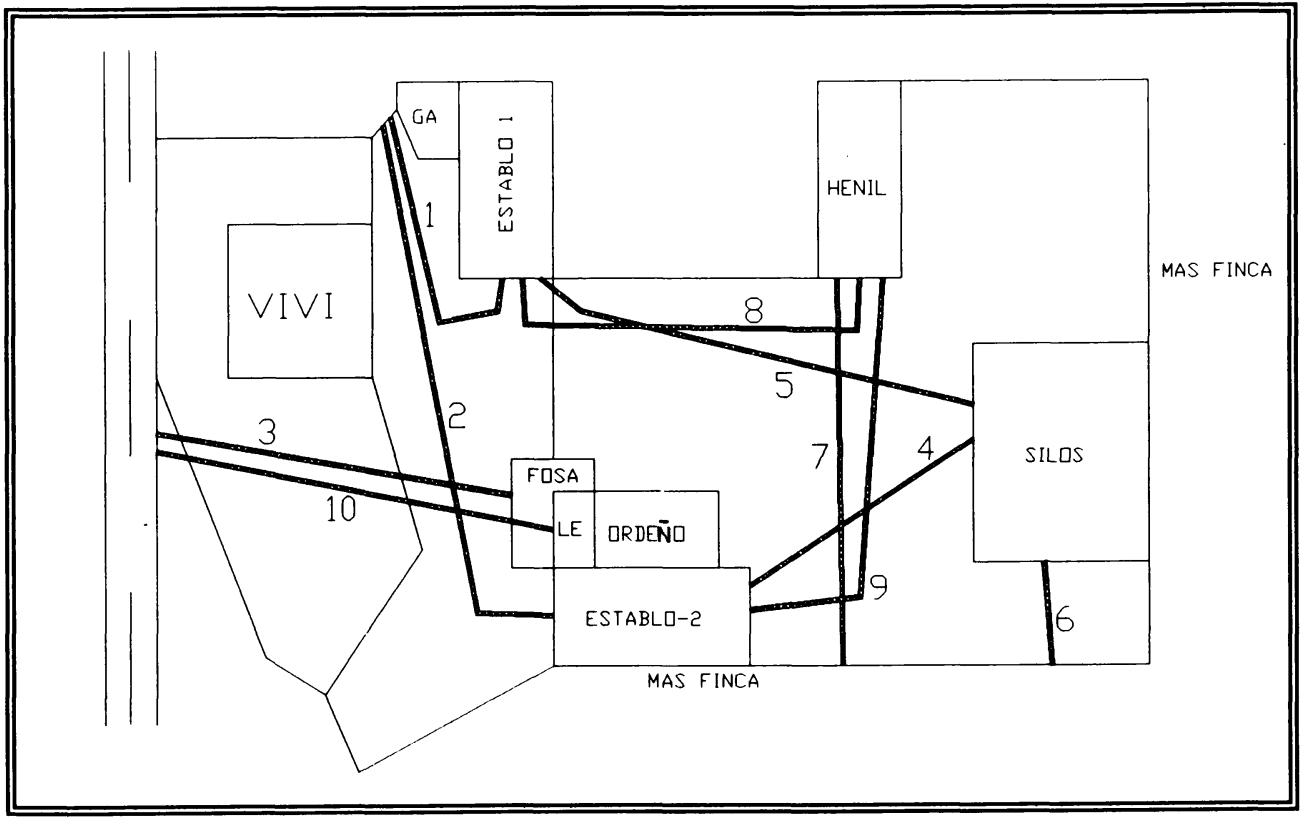

Figura 2.- Determinación de parámetros de distancias. 


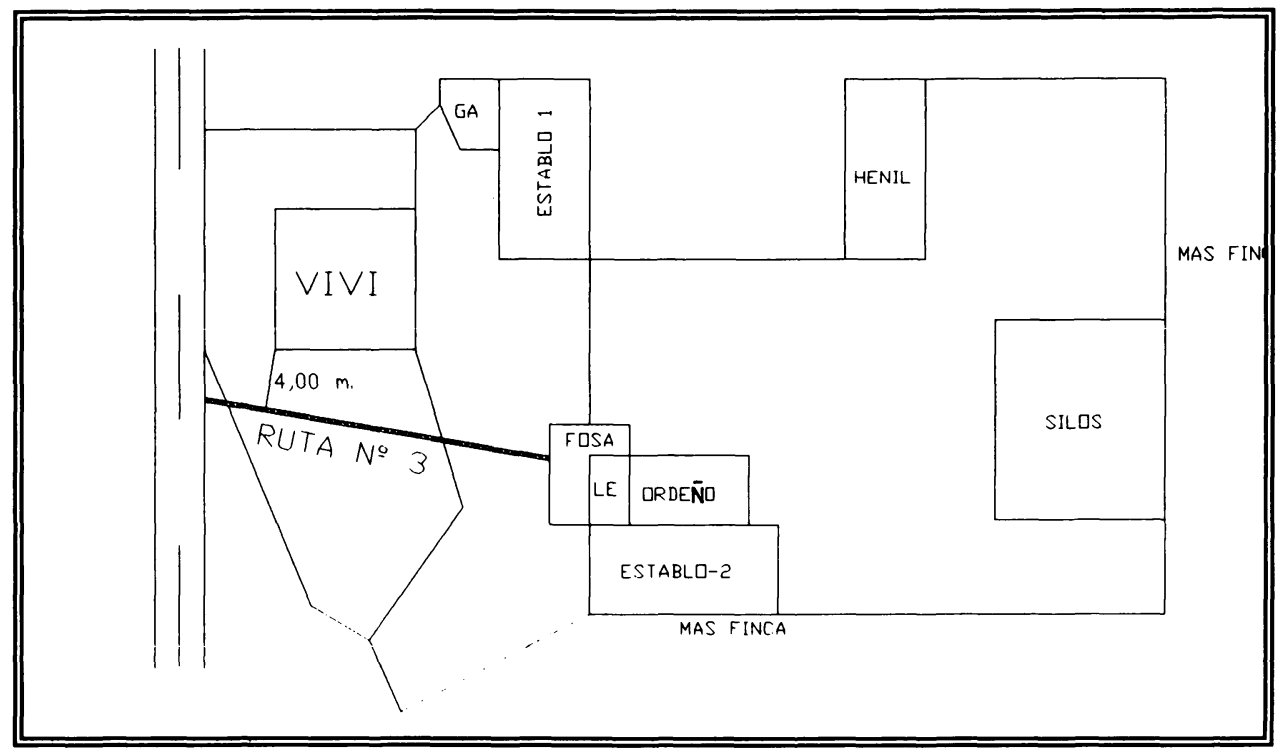

Figura 3.- Determinación de distancias a la vivienda.

En donde se indica que la distancia mínima, desde la ruta $\mathrm{n}^{0} 3$ hasta la vivienda, es de 4,00 metros.

\subsubsection{El tratamiento de los datos}

Las variables iniciales del sistema son 94 , de las cuales, 78, son cuantitativas y 16 cualitativas.

Fue necesario identificar previamente aquéllas que pudieran aportar información, para hacerlas intervenir en el análisis y eliminar las variables redundantes o aquéllas entre las que se observó una clara dependencia lineal, ya que podían falsear los análisis posteriores. Para ello se realizó un análisis de la varianza de las variables cuantitativas sobre las cualitativas, considerando modelos de un solo factor, cada variable cualitativa y tantos niveles como clases presentaba la variable.

En cada caso se empleó un modelo de efectos fijos, dado que la heterogeneidad del conjunto de explotaciones muestreado no permite afirmar a priori que constituyen un grupo homogéneo de varianza común.

A continuación se realizó un estudio de correlación en el que intervinieron todas las variables cuantitativas que habían dado efectos significativos en alguno de los análisis anteriores. Se detectaron linealidades y variables redundantes, que fueron eliminadas en los análisis posteriores, con lo que el número de variables cuantitativas tenidas en cuenta fue de 52.

Posteriormente se realizó un análisis de componentes principales (ACP) sobre dichas variables. Se identificaron aquellas variables iniciales relacionadas con las componentes principales de mayor autovalor asociado.
Finalmente, se representaron las explotaciones en los primeros planos factoriales.

La influencia de las variables cualitativas sobre el conjunto de las cuantitativas se trató de identificar, representando las clases en los planos factoriales.

A continuación se realizó una clasificación ascendente jerárquica, empleando la distancia euclídea estándar y la estrategia de clasificación de Ward.

Para la clasificación se emplearon las coordenadas de las explotaciones en las cuatro primeras componentes principales, lo que permitió retener una gran cantidad de información y eliminar ruido aleatorio.

A la vista del árbol de clasificación y de la inercia explicada se realizó una partición del conjunto de explotaciones.

Las clases obtenidas se representaron en los primeros planos factoriales y se describieron en función de las variables iniciales. Para ello se utilizaron las relaciones entre éstas y las componentes principales.

Los resultados se confirmaron mediante un nuevo análisis de la varianza de las variables cuantitativas considerando como factor de clase y un test de Duncan posterior para probar la diferencia entre las medias de clase.

\section{RESULTADOS}

Realizado el análisis de componentes principales sobre las variables cuantitativas, se han obtenido los siguientes resultados (Tabla 4): 
TABLA 4

Componentes principales

\begin{tabular}{||c|c|c|l||}
\hline Componente principal & Autovalores & Inercia acumulada & \multicolumn{1}{|c|}{ Variables relacionadas } \\
\hline 1 & 8,7842 & 0,1689 & NRUT, DGAN, SPRI, DHEN, \\
2 & 5,0502 & 0,2660 & DPUR, STOT, NINCUM, \\
3 & 4,3078 & 0,3488 & IRUT, SVEG, RTR, NHVP, \\
4 & 2,9779 & 0,4061 & SASEP, NBVP, \\
5 & 2,5079 & 0,4543 & VLECH, BT \\
6 & 2,0895 & 0,4945 & LECHS, MIEM \\
7 & 2,0245 & 0,5335 & \\
8 & 1,7037 & 0,5662 & \\
9 & 1,6018 & 0,5970 & \\
10 & 1,4835 & 0,6256 & \\
11 & 1,4086 & 0,6527 & \\
12 & 1,3344 & 0,6784 & \\
13 & 1,2922 & 0,7032 & \\
14 & 1,1559 & 0,7254 & \\
15 & 1,0981 & 0,7466 & \\
16 & 1,0291 & 0,7664 & \\
\hline
\end{tabular}

Los autovalores vienen a indicar la inercia (varianza multidimensional) explicada por la componente principal asociada. Dado que cada variable inicial tiene varianza igual a 1 (centrada y reducida), las componentes principales con autovalores menores a la unidad carecen de interés, pues explican menos variabilidad que la derivada de las variables iniciales.

En consecuencia, hemos seleccionado las variables con autovalores superiores a 1 .

Se observa, pues, que las componentes consideradas explican, en conjunto, una fracción de la inercia total apreciable, del $76,64 \%$

Elanálisis de componentes principales confirma los criterios utilizados para la agrupación de las variables iniciales, observándose que la primera componente se asocia con variables del grupo "rutas y circulaciones"; la segunda con las del grupo "Parcela-base" y, la tercera, con las del grupo "vivienda".

Por otra parte, la inercia explicada por las siete primeras componentes principales es mayor del $50 \%$, valor bastante alto teniendo en cuenta el muy elevado número de variables estudiadas.

La primera componente principal explica el $16,89 \%$ de la inercia total, pudiendo ser interpretado como un eje de actividad agraria.

La segunda componente principal se puede interpretar como un eje en el que se representan valores de características de la Parcela-base.
La tercera presenta altas correlaciones con la variables del grupo "vivienda", por lo que se podrá considerar como un eje de calidad de la vivienda.

La cuarta componente principal se asocia con factores relacionados con la capacidad productiva de la explotación.

\section{CONCLUSIONES}

El sistema "Vivienda familiar-Explotacion Agraria" en Galicia presenta una importante variabilidad.

Para la cuantificación de la calidad de vida en el sistema, las características de la vivienda y el nivel de renta percibido en la explotación tienen una importancia menor de la que, en principio, se podría suponer.

Las variables del sistema, que inciden sobre la calidad de vida, ordenadas en función de su importancia, son las siguientes:

1. Actividad agraria en la Parcela-base (Componente Principal $n^{\circ}$ 1)

2. Características de la Parcela-base (Componente Principal no 2)

3. Características de la vivienda (Componente Principal $\mathrm{n}^{\circ} 3$ )

4. Capacidad productiva de la explotación (Componente Principal $\mathrm{n}^{\circ}$ )

Mediante la utilización de las variables antes referidas se 
han podido clasificar las explotaciones de la muestra en cinco clases bien diferenciadas:

Clase A: explotaciones de baja capacidad productiva con unas condiciones muy deficientes de la parcela-base y de la vivienda

Clase B: alta capacidad productiva, muy deficiente distribución de los edificios y buenas características de la parcela-base.

Clase C: similar a la clase A, con la diferencia de que mejoran las características de la parcela-base, la vivienda y las actividades agrarias.

Clase D: capacidad productiva muy baja, con viviendas antiguas de grandes dimensiones.

Clase E: alta capacidad productiva con separación de la vivienda de la parcela en la que se ubican las instalaciones ganaderas.

De acuerdo con el propio planteamiento de la investigación se abre la posibilidad de realizar nuevos estudios sobre el sistema "Vivienda Familiar-Explotación Agraria" con la consideración de las variables que definen su nivel de calidad de vida

Los parámetros de diseño más importantes son:

1. Concentración de los alojamientos ganaderos en una zona concreta $\mathrm{y}$, si fuera posible, dentro de una única edificación.

2. Ubicación de los alojamientos ganaderos, fosa de purín y silos a una distancia mínima de la vivienda, que habría que establecer, mediante el correspondiente estudio.

3. Delimitar claramente una zona para la circulación de vehículos, maquinaria y ganado, dentro de la parcela-base, acondicionándola debidamente.

4. Establecer una superficie mínima para la parcela-base, en función de las características de la explotación.

5. Determinar en que forma se deberá acondicionar el entorno de la vivienda y en qué radio mínimo alrededor de la misma.

\section{BIBLIOGRAFÍA}

- BLANCO DOPICO, A.: 1979. "Medio geológico y arquitectura rural en S. Martín de Castrelo, Vimianzo (La Coruña)". Gallaecia. 3-4. pp. 357-366. Dpto. de Prehistoria y Arqueología. Santiago.

- BOUHIER, A.: 1979. “La Galice. Essai geographique d'analyse et d'interpretation d'un vieux complexe agraire". La Roche-SurYon (vendee). Imprierie Yonnaise.
- CARREIRA PEREZ, X.C.: 1996. "Aloxamentos para gando vacún de leite”. Xunta de Galicia. Santiago.

-CASABELLA,X.: 1984. "Xornada sobor da vivenda unifamiliar en Galicia”. Obradoiro.- n' 9.- abril. pp. 50-53. C.O.A.G. La Coruña.

- CLELAND, D.I.; KING, W.R.: 1981. "Systems analysis and project management”. McGraw-Hill. New York.

- CONSELLERÍA DE AGRICULTURA, GANDEIRIA E MONTES.: 1995. “Anuario de Estadística Agraria. 1994". Consellería de Agricultura. Xunta de Galicia. Santiago.

- CUADRAS, C.M. 1996.: "Métodos de Análisis Multivariante". EUB, S.L. Barcelona.

- DAMM, T.: 1979. “Umbau alter Bäuernhauser in NordrheinWestfalen”. Landtechnik, 1 Januar. pp. 18-21.

- DAMM, T.: 1982. "Landschaftsbezogenes bauen in Nordrhe Westfalen”. Landtechnik v 37 (12), pp. 540-541.

- DAMM, T.: 1988. "Landwirtschaftliches bauen in der Landschaft". Landtechnik, 10 vol. 43, pp. 428-430.

- DE LLANO CABADO, P.: 1983. "Arquitectura popular en Galicia” (Tomo II). C.O.A.G. La Coruña.

- DE LLANO CABADO, P.: 1989. “Arquitectura popular en Galicia” (Tomo I). C.O.A.G. La Coruña.

- DEL CANTO FRESNO, C.: 1992. "Desarrollo rural. Ejemplos europeos”. M.A.P.A.- IRYDA. Madrid.

- DOYAL, L; GOUGH, I.: 1994. "Teoría de las necesidades humanas". Icaria. Fuhem. Barcelona.

- ERICKSON, R.; ABERG, R.: 1987. "Welfare in transition: a survey of living conditions in Sweden (1968-1981)". Clarendon Press. Oxford.

- ETXEZARRETA, M.; CRUZ, J.; GARCÍA MORILLA, M.; VILADOMIU, L.: 1995. "La agricultura familiar, ante las nuevas políticas agrarias comunitarias”. S.G.T.- M.A.P.A. Madrid.

- FONDATION RURALE DE WALLONIE.: 1984. "Fiches d'aménagement Rural". Ministère des Travaux Publics. Bruxelles.

- FORTIER, B.; VAYSSIERE, B.: 1980. "L'architecture des Villes. Espaces, cartes et territoires”. Urbi. III

- GARCÍA FERNÁNDEZ, J.L.; PRIETO GRANDA, F. et al.: 1984. "Patrimonio Urbanístico, Arquitectónico y Arqueológico del Corredor Madrid Guadalajara". Centro de información y documentación. Comunidad de Madrid.

- GARCIIA GRINDA, J.L.: 1991. “Arquitectura PopularLeonesa”. Excma. Diputación Provincial de León. León.

- GÓMEZ OREA, D.: 1994. "Ordenación del Territorio. Una aproximación desde el Medio Físico". Agrícola Española, S.A. Madrid. 
- KOEBEL C.T.; ENGELEN-EIGLES, D.A.; CAVELL, M.S.: 1991. "Rural Housing trends in Virginia: a profile of the eighties". Virginia Center for Housing Research. Virginia. U.S.A.

- L'ASSISTANCE ARCHITECTURAL ET URBANISTIQUE, 1995. "Les Portes de Grange" ( $2^{\mathrm{a}}$ edition). Fondation Rurale de Wallonie. Attert.

- L'ASSISTANCE ARCHITECTURALE ETURBANISTIQUE.: 1995. "Les abords de la maison". Fondation Rurale de Wallonie. Attert.

- M.W.P.S.: 1990. "House planning handbook". M.W.P.S. IowaU.S.A.

- MACKAY, S.: 1979. "Home storage of fruits and vegetables". N.R.A.E.S. Ithaca.

- MACKAY, S.: 1980. "Home Heating in an emergency". N.R.A.E.S. Ithaca.

- MACKAY, SUSAN et al.: 1985. "Burning wood and coal". N.R.A.E.S. Ithaca.

- MANCL, K.; SAILUS, M; WAGENET, L.: 1991. "Private drinking water supplies (quality, testing, and optio)". N.R.A.E.S. Ithaca.

- OLSON, E.A.; JOHNSON, D.J. etal.: 1982. "Farmstead Planning Handbook". MWPS. Ames. Iowa. USA.

- ORJALES PITA, M.: 1975. "Crise da Casa como Sistema de Producción e máis de Convivencia”. Ed. Galaxia. Vigo.

- PORTELA, C.: 1984. "Aportaciones para un debate de la Vivienda Unifamiliar en el medio rural”. Obradoiro. $n^{\circ} 9$. pp. 2836. C.O.A.G. La Coruña

- RABANOS FACI, C. et al.: 1993. "La Casa Rural en el Pirineo Aragonés". Diputación de Huesca. Instituto de Estudios Alto Aragoneses. Huesca.

- ROMERO VILLAFRANCA,: 1989. "Técnicas de Análisis de Datos Multidimensionales”. M.A.P.A. Madrid

-S.G.T. M.A.P.A.: 1990. "Políticas Administrativa y Económica de la Colonización Agraria. Análisis Institucional y Financiero". M.A.P.A. Madrid.

- SAMARANCH SALISACHS, M.T.: 1989. “Análisis Económico Financiero de la Vivienda: un enfoque Empresarial”. Tesis Doctoral. Universitat de Barcelona. Barcelona.
- SANTOS, G.: 1984. "Xornada sobor da vivenda unifamiliar en Galicia”. Obradoiro.- ${ }^{\circ}{ }^{9}$ 9.- abril. pp. 50-53. C.O.A.G. La Coruña.

- SANZ JARQUE, J.J.: 1982. “Aspectos sociológicos sobre la explotación familiar en España". Explotaciones Familiares Agrarias y Agricultores Jóvenes. Estudios sobre el Proyecto de Estatuto. pp. 35-48. Publicaciones de Extensión Agraria. Madrid.

- SHUCKSMITH, M.: 1991. "An assessment of crofter housing improvement policies”. Scottish Geografical Magazine. vol. 107. $n^{\circ} 1$. pp. 33-39.

- SHUCKSMITH, M.; HENDERSON, M.; RAYBOULD, S. et al.: 1995. "A classification of rural housing markets in England". Hmso. London.

-SHUCKSMITH, M.; WATKINS, L.; HENDERSON, M.: 1993. "Attitudes and policies towards residential development in the scottish countryside". Journal of Rural Studies. Vol. 9. $n^{\circ} 3$. pp. 243-255. Pergamon Press ltd. Aberdeen.

- SHUCKSMITH, M.: 1988. "Policy aspects of housebuilding on farmland in Britain. Land development studies". pp. 129-138. Land Development Studies Education Trust. Aberdeen.

- SHUCKSMITH, M.: 1990. "Rural Housing in the UK. Current Policy Issues”. Land Use policy. October. pp. 283-286. Viewpoint. Aberdeen.

- SHUCKSMITH, M.; WATKINS, L.: 1991. "Housebuilding on Farmland: The distributional effects in rural areas". Journal of Rural Studies. Vol. 7. $n^{\circ}$ 3. pp. 153-168. Pergamon Press, plc. Great Britain.

\section{REFERENCIAS BIBLIOGRÁFICAS:}

(1) DOYAL, L.; GOUGH, I.:1994. "Teoría de las necesidades humanas". Icaria. Fuhem. Barcelona.

(2) GÓMEZ OREA, D.: 1994. "Ordenación del territorio. Una aproximación desde el medio físico". Agrícola Española, S.A. Madrid.

(3) CONSELLERIA DE AGRICULTURA (Xunta de Galicia): 1995. “Anuario de estadística agraria. 1994". Xunta de Galicia.

(4) CLEALAND, D.I.; KING, W.R. : 1981. "Systems analysis and project management". McGraw-Hill. New York. 